\title{
Asessing public policies. The case of education in Europe and the interaction between personal and institutional factors
}

\author{
Clara Riba \\ Departament de Ciències Polítiques i Socials \\ Universitat Pompeu Fabra \\ Anna Cuxart \\ Departament d'Economia i Empresa \\ Universitat Pompeu Fabra \\ clara.riba@upf.edu, anna.cuxart@upf.edu
}

Keywords: education, public opinion, public policies, multilevel analysis, comparative studies, European Social Survey

Palabras clave: educación, opinión pública, políticas públicas, análisis multinivel, estudios comparados, Encuesta Social Europea

Journal of Economic Literature Classification: C42 


\title{
Resumen
}

El presente trabajo aborda el estudio comparado del nivel de satisfacción de los ciudadanos europeos con el estado de la educación contrastando la existencia de efectos individuales y de efectos derivados de las distintas políticas educativas aplicadas. Se aplica una metodología multinivel para contrastar la existencia de interacciones entre los ámbitos individual y agregado. Los datos muestran que las decisiones políticas de los gobiernos (el grado de descentralización educativa, el grado de inclusión del sistema y el nivel de gasto público en educación) así como el entorno social de los estudiantes (nivel socioeconómico y cultural familiar medio) influyen significativamente en las opiniones acerca del estado de la educación que tienen los ciudadanos.

\begin{abstract}
The paper deals with the comparative study of European citizens' satisfaction with the state of education in their respective countries. Individual and contextual effects are tested applying multilevel analysis. The results show that educational public policies (level of decentralization, degree of comprehensiveness and public spending) as well as the students' social environment (socioeconomic and cultural status) have a sound impact on the opinions about the state of education.
\end{abstract}




\section{Introducción}

La política educativa constituye una de las políticas públicas fundamentales de los estados de bienestar. En Europa, la educación ha sido tradicionalmente concebida como una herramienta para la promoción de la igualdad de oportunidades entre ciudadanos y es considerada un elemento clave del actual proceso de construcción europea. La Unión Europea reconoció el papel crucial que la educación juega en el progreso de las sociedades cuando, en el Consejo Europeo de Lisboa de marzo del año 2000, fijó como objetivo estratégico convertir a Europa en una sociedad basada en el conocimiento y la economía, capaz de crecer económicamente de manera sostenible con más y mejores empleos y con mayor cohesión social. Complementariamente, la Declaración de Bolonia, por la que los países firmantes se comprometen en la creación de un Espacio Europeo de Educación Superior común para el año 2010, obliga a los gobiernos a replantearse su educación superior poniendo especial énfasis en la formación permanente y en las estrategias para "aprender a aprender" durante toda la vida. Pero no son sólo las políticas universitarias las que importan. La educación de los ciudadanos es un proyecto colectivo de la sociedad, cuyo reto es el de formar ciudadanos capaces de afrontar las dinámicas aceleradas de cambio manteniendo, sin embargo, la cohesión social. Según el expresidente de la Comisión Europea Jacques Delors, una educación de calidad debe ser capaz de atender de manera integrada las demandas crecientemente heterogéneas de las sociedades actuales (Delors, 1996). Y es en este contexto que es de interés conocer la opinión de los ciudadanos sobre cómo responden los gobiernos al reto de la educación en los inicios del siglo XXI.

La opinión pública, en tanto que medida agregada de las preferencias individuales, es el instrumento que permite contrastar la satisfacción de la ciudadanía con las medidas implementadas (Dalton, 1989). El conocimiento por parte de los gobiernos de las demandas de sus ciudadanos así como la constatación del grado de satisfacción de los mismos con las respuestas dadas requiere, pues, del análisis de la opinión pública. La opinión pública es un concepto plenamente incorporado en el análisis político y los estudios de opinión pública realizados en las últimas décadas son numerosos, constituyendo hoy en día una rama importante de la literatura de la disciplina. Los trabajos publicados muestran la consistencia y estabilidad de la opinión pública (Page y Shapiro, 1992), el encaje entre información y valores en la formación de las opiniones 
individuales (Zaller, 1991; Page y Shapiro, 1992), el rol que juegan los medios de comunicación en este proceso (Page, 1996; Pallarès y Gifreu, 1998) y, en especial, la influencia de la opinión pública en las políticas de los gobiernos (Page y Shapiro 1983; Stimson, Mackuen y Erikson 1995).

Las encuestas de opinión son el principal instrumento de medida de la opinión pública. Desde 1944, año en que se realizó el primer estudio por encuesta a gran escala (Lazarsfeld, Berelson y Gaudet, 1944), hasta nuestros días la metodología de las encuestas ha evolucionado enormemente. Así, el diseño muestral que aplica la teoría de la probabilidad aporta la base científica para la realización de inferencias (Kish, 1965) ${ }^{1}$ mientras que la investigación sobre la tipología de preguntas y respuestas permite afinar cada vez más el instrumento de medida (Saris, Satorra and Coenders; 2004; Saris, vander Veld and Gallhofer, 2004). Sin embargo, persisten una serie de prácticas deficientes en muchas de las encuestas que se realizan actualmente que ponen en cuestión la calidad de las medidas que de ellas se obtienen (Riba y Cuxart, 2003; Cuxart y Riba, 2005). De todo ello se deriva que la medida de la opinión pública puede variar de manera apreciable en función de la calidad de la metodología aplicada en la realización de las encuestas.

El objetivo de este estudio es el conocimiento del nivel de satisfacción ciudadana con la educación, su variabilidad entre países y los posibles factores que inciden en dicha satisfacción, utilizando para ello datos de la Encuesta Social Europea (ESE) y datos por países procedentes de la OECD.

\section{Modelo explicativo de la satisfacción con la educación}

Se parte del supuesto que hay dos tipos de factores que afectan la opinión de los ciudadanos acerca del estado de la educación en su país. Los unos están relacionados con las características de cada individuo y varían de un individuo a otro, mientras que los otros están relacionados con el entorno en el que vive el ciudadano. Este segundo

\footnotetext{
${ }^{1}$ En 1936 George Gallup mostró el valor de las técnicas probabilísticas aplicadas al diseño de muestras al predecir el triunfo de Franklin D. Roosevelt en las elecciones presidenciales, contra su oponente Alfred M. Landon a quien otro sondeo patrocinado por el Literary Digest daba como triunfador (Squire, 1988).
} 
grupo de factores hacen referencia al contexto social y político de cada país e interaccionan con los factores individuales. A continuación se exponen cuáles son los factores individuales y contextuales que se toman en consideración, explicitando en cada caso el mecanismo causal que los conecta con la variable dependiente.

\section{Factores individuales: hipótesis}

¿Por qué dentro de un mismo país hay unos ciudadanos que valoran bien la educación y otros que la valoran mal? En primer lugar, es razonable suponer que las circunstancias individuales de las personas les llevan a tener opiniones distintas. Variables de tipo socio estructural como el sexo, la edad o el nivel de estudios alcanzado explican en parte estas diferencias. Así, en primer lugar, las mujeres, por su pertenencia a un grupo social en general más vulnerable y por su tradicional rol de atención a niños y ancianos, tienden a dar mayor soporte a las políticas de protección social que los hombres. En segundo lugar, se espera que los jóvenes tengan posiciones más extremas (en positivo o en negativo) que las de la media de los ciudadanos, debido a la cercanía temporal de su propia experiencia educativa. Los individuos con un alto nivel de estudios, por su parte, se espera que sean más críticos con el estado de la educación en sus países que los que tienen un nivel de estudios más bajo como consecuencia de su mayor conocimiento del tema y de su mayor capacidad analítica. Estudios empíricos muestran, efectivamente, la existencia de diferencias por género y edad en la valoración de las políticas públicas (Schlesinger y Heldman, 2001; Blekesaune y Quadagno, 2003) y de niveles de satisfacción menores entre los individuos con mayor nivel de educación en España (Fraile, 2005). Por lo tanto, se espera que:

- existan diferencias en las valoraciones de hombres y mujeres.

- las valoraciones de los jóvenes sean más extremas que las de los mayores.

- las valoraciones de los más educados sean más bajas que las de los poco educados.

En segundo lugar, la perspectiva ideológica también juega su papel en las opiniones. En general, la identificación política y la proximidad con el partido o coalición en el poder influyen en la valoración de las políticas del Gobierno. En este sentido, el ciudadano que vea con preocupación el estado de la educación en su país pero que esté próximo al Gobierno puede matizar su valoración negativa por tener expectativas claras de mejora 
y sentirse copartícipe de las actuaciones gubernamentales. En sentido contrario, un ciudadano que esté próximo a la oposición puede tener incentivos para evaluar negativamente las políticas que surjan del Gobierno, entre ellas la política educativa aunque la considere razonablemente buena, con el objetivo de desgastar el equipo en el gobierno. En consecuencia, las evaluaciones del estado de la educación pueden estar contaminadas por la identificación y/o proximidad del individuo con los partidos del gobierno o con los partidos de la oposición. Así, pues, en el análisis de las causas de la satisfacción con la educación parece conveniente introducir como variable de control la proximidad o identificación del individuo con los partidos gobernantes en cada país ${ }^{2}$.

\section{Factores contextuales: hipótesis}

Una vez analizadas las posibles causas de la existencia de variaciones en las valoraciones dentro de un mismo país, la pregunta clave que se plantea es: ¿Por qué, en promedio, la valoración de los ciudadanos de un país es distinta de la de los de otro? Aquí parece lógico suponer que se deban a la existencia de distintas políticas educativas y a la presencia de diferencias socioculturales de su población. Entre las variables que reflejan políticas educativas tienen especial importancia, entre otras, el volumen de gasto público en educación, el grado de descentralización de las decisiones en materia educativa, el grado de inclusión del sistema, determinados aspectos de la estructura del sistema educativo, así como las políticas de profesorado.

El volumen de gasto público en educación es uno de los indicadores de la importancia que los gobiernos dan a este tema. Una educación de calidad requiere de presupuestos adecuados para su implementación y, aunque la disposición de fondos abundantes no garantiza la calidad, la falta de recursos suficientes sí es un obstáculo para la misma. Por otra parte, tal como se ha comentado en la introducción, una educación de calidad debe ser capaz de atender de manera integrada las demandas crecientemente heterogéneas de las sociedades actuales (Delors, 1996). Y esto parece difícil conseguirlo con una

\footnotetext{
${ }^{2}$ El indicador utilizado es la valoración de la gestión del gobierno. Ciertamente, su inclusión como variable de control en el modelo explicativo de la valoración de la educación puede plantear un cierto problema de circularidad, pero los datos revelan que la correlación entre ambas variables es relativamente baja (0,38 para el conjunto de países y 0,36 para los datos de España). Por otro lado otros indicadores alternativos como "recuerdo de voto" o " proximidad ideológica" presentan problemas similares.
} 
organización centralizada y excesivamente rígida, ya que es desde la proximidad desde donde se pueden diseñar y adaptar mejor las políticas a las necesidades de cada entorno (Subirats, 2002; Bonal, 1998) ${ }^{3}$. Diversos estudios empíricos subrayan el valor de la descentralización por su contribución a la resolución de problemas complejos, a la modernización y a la mejora del nivel de vida (Ortún y López-Casanovas, 2000; Llera, 2003). En ese sentido, el grado de descentralización de las políticas educativas es un buen indicador de la capacidad de respuesta del sistema a las demandas de la sociedad. Por lo que respecta al grado de inclusión del sistema, aquellos países que tengan sistemas más comprensivos admitirán en su seno alumnos con una gran diversidad de aptitudes y capacidades, buscando las adaptaciones necesarias para que todos los alumnos puedan progresar en su educación. Probablemente, en términos generales, el nivel de satisfacción de la ciudadanía será más elevado en esos países que en otros con un sistema más selectivo que excluye de su seno a los alumnos que no son capaces de superar en su momento los niveles establecidos. En ese sentido, el informe PISA 2003 subraya los logros educativos de países con sistemas inclusivos (OECD, 2004). Se puede argumentar que existen otros aspectos, como la edad de inicio de la escolarización obligatoria, su duración o la existencia de un exámenes de final de ciclo, que tienen también efecto en la opinión de los ciudadanos. Sin embargo no existe un consenso generalizado sobre la dirección de esos efectos ni se ha hallado evidencia empírica que los confirme, motivo por el cual en el presente trabajo no se plantean hipótesis en ese sentido. Por lo tanto, en referencia a aspectos de política educativa, se espera que la satisfacción con el estado de la educación sea más elevada en los países:

- que inviertan proporcionalmente más recursos en educación.

- donde haya un mayor grado de descentralización de las decisiones educativas.

- que posean un sistema educativo más inclusivo.

El que sí que se ha revelado como un aspecto determinante de la calidad de la educación es el prestigio que otorga la sociedad a la profesión docente ${ }^{4}$. Una educación de calidad requiere de la presencia de profesionales bien formados, motivados, que se sientan

\footnotetext{
${ }^{3}$ Sin embargo, algunos expertos alertan del peligro de introducción de desigualdades territoriales que puede entrañar una descentralización total de las políticas educativas (Gairin, 2005).

${ }^{4}$ Un estudio sobre los factores de éxito del sistema educativo finlandés, país que en el estudio PISA 2003 alcanzó los mejores resultados, destaca la alta consideración social y la sólida formación de sus docentes (Consell Superior d’Avaluació del Sistema Educatiu, 2005).
} 
socialmente valorados y que dispongan de medios para realizar adecuadamente su trabajo. Se espera, pues, que:

- a mayor prestigio social de la profesión docente mayor satisfacción con la educación

Ante la dificultad de hallar indicadores de la valoración social del docente, en el presente trabajo se optó por utilizar un conjunto de variables relacionadas con las condiciones laborales del profesorado (salario inicial y carrera, ratio alumnos/profesor ${ }^{5}, \ldots$ ) así como otros indicadores proxi de prestigio (feminización de la profesión docente ${ }^{6}$ ) como variables explicativas de la satisfacción con la educación.

Además de las decisiones del gobierno, influye también en la calidad de la educación el contexto socioeconómico en el que se desarrolla la tarea formativa. Parece claro que aquellos alumnos que tengan un entorno familiar preocupado por la educación, que dispongan de libros y materiales formativos y que tengan acceso a nuevas tecnologías sacarán más provecho de las actividades formativas que aquellos que se desenvuelvan en entornos poco propicios al estudio y que carezcan de dichos recursos. Así, partiendo de que el estatus socioeconómico y cultural de las familias de los alumnos incide positivamente en los resultados que éstos obtienen (OECD, 2004) y que los buenos resultados académicos de los alumnos afectan positivamente las percepciones ciudadanas acerca de la calidad de la educación, es razonable suponer que el nivel socioeconómico y cultural medio de las poblaciones de los países explique en parte las diferencias de sus valoraciones acerca de la educación. Por otra parte, los resultados del proceso educativo serán mejores y el nivel de satisfacción de las familias será más alto si los propios alumnos se implican en su educación. En aquellas sociedades donde los alumnos se implican en las actividades de sus centros educativos y desarrollan sentimientos de pertenencia hacia sus respectivas comunidades, los niveles de satisfacción de estudiantes, profesores y padres con la educación son elevados (Subirats,

\footnotetext{
${ }^{5}$ Un número reducido de alumnos por aula permite una mayor interacción profesor-alumno, facilita una atención personalizada, y permite la aplicación de una diversidad de metodologías. Por lo tanto ratios reducidas deberían facilitar la labor del docente al mismo tiempo que aumentar la satisfacción con la educación. El estudio PISA 2003 no detectó asociación entre ratios de alumnos/profesor y resultados académicos, aunque dicho estudio sugiere que la falta de asociación se debe posiblemente a que es en los grupos reducidos donde se suelen escolarizar los alumnos con necesidades educativas especiales y rendimientos más bajos que la media.

${ }^{6}$ Se entiende que la existencia de porcentajes equilibrados de hombres y mujeres en los colectivos de docentes es un signo de valoración social de la profesión.
} 
2002; OECD, 2004) ${ }^{7}$. Se espera, por lo tanto, que las valoraciones sobre el estado de la educación sean más altas:

- en los países donde el estatus socioeconómico y cultural medio de las familias de los estudiantes sea más elevado.

- en las sociedades donde hay un mayor nivel de participación e implicación del alumnado en su propia educación.

\section{Metodología y datos}

Las opiniones de los ciudadanos europeos ${ }^{8}$ sobre el estado de la educación en sus respectivos países, son analizadas combinando la información individual de la Encuesta Social Europea (ESE) del $2002^{9}$ con datos del contexto social e institucional de los países participantes en el estudio ${ }^{10}$. Los datos de la ESE, obtenidos con una metodología que tiene unos estándares de calidad muy superiores a los de las encuestas habituales, proporcionan una sólida base de datos para el análisis, permiten la comparabilidad entre países europeos y posibilitan el análisis de la evolución de las opiniones a lo largo del tiempo $^{11}$. La estimación multinivel aplicada permite contrastar la existencia de interacciones entre los ámbitos individual y agregado de manera eficiente.

La variable dependiente o respuesta es la valoración del estado de la educación en el propio país. Las variables explicativas usadas en el análisis, tanto las de tipo individual

\footnotetext{
${ }^{7}$ Los resultados de una serie de estudios empíricos sobre educación y territorio en España indican que la satisfacción con el estado de la educación aumenta cuando en los centros educativos se crean comunidades identificadas con sus respectivos proyectos y que el sentido de comunidad aparece cuando existe la participación y la implicación de sus miembros en el desarrollo de las actividades formativas.

${ }^{8}$ Por ciudadanos europeos se entiende el conjunto de ciudadanos de los países participantes en la primera ola de la Encuesta Social Europea: Austria (AT), Bélgica (BE), Suiza (CH), República Checa (CZ), Alemania (DE), Dinamarca (DK), España (ES), Finlandia (FI), Francia (FR), Reino Unido (GB), Grecia (GR), Hungría (HU), Irlanda (IE), Israel (IL), Italia (IT), Luxemburgo (LU), Holanda (NL), Noruega (NO), Polonia (PL), Portugal (PT), Suecia (SE) y Eslovenia (SL).

${ }^{9}$ En el momento de cerrar el análisis de datos de este trabajo han sido publicados los datos de la ola 2004 de la ESE para un grupo de países. Ello ha permitido replicar para los datos de 2004 el mismo análisis que se realiza con los datos de 2002 y comprobar que los resultados son coherentes. En el Anexo 1 se presentan dichos resultados.

10 La fuente de datos es la OECD, siendo la mayor parte de variables procedentes del estudio PISA 2003, pero también del estudio Education at a Glance 2004.

${ }^{11}$ La bondad de la metodología de la ESE no evita, no obstante, el posible sesgo derivado de la información recibida por los ciudadanos ya que, en palabras de Page, si la información es incompleta, incorrecta, engañosa o declaradament falsa, entonces la gente puede incluso favorecer políticas que le son perjudiciales (Page, 1996).
} 
como las de tipo contextual, han sido escogidas con el objetivo de contrastar las hipótesis que se derivan del modelo explicativo de la satisfacción con el estado de la educación expuesto en el apartado anterior. Éstas son:

- Variables individuales: Sexo, edad, nivel de estudios y proximidad al gobierno.

- Variables contextuales: Gasto público en educación, grado de descentralización de las decisiones educativas ${ }^{12}$, grado de inclusión del sistema $^{13}$, ratios profesor/alumno en primaria y secundaria, salario inicial de los profesores y salario a los quince años, relación entre salario a los 15 años y el PIB por cápita, volumen de la formación continua, porcentaje de mujeres entre los docentes de primaria y secundaria, índice socioeconómico y cultural del país ${ }^{14}$, porcentajes de estudiantes con baja implicación y bajo sentido de pertenencia a su centro educativo.

El análisis realizado consta de tres etapas. En la primera etapa se ha realizado una descripción de las valoraciones medias del estado de la educación para el conjunto de los 22 países participantes en la Encuesta Social Europea. En la segunda etapa se ha estimado un modelo de variación de la variable respuesta de dos niveles con las variables explicativas individuales. Este procedimiento ha permitido seleccionar del conjunto de variables explicativas individuales consideradas inicialmente aquellas que han mostrado ser más significativas. En la tercera etapa se han introducido en el modelo las variables contextuales.

Ante la dificultad de encontrar información sobre las variables de política educativa de los 22 países, se ha optado por realizar el análisis por separado para dos grupos distintos de países. En unos modelos se ha estudiado el papel de las variables contextuales gasto y descentralización, de las cuales se dispone de información para un grupo de 17 países. En otros, se ha analizado el papel de la variables de inclusión del sistema e índice socioeconómico y cultural, de las que se tiene información para otro grupo de 17 países (que coinciden en parte pero no totalmente con los anteriores). Así, con esta estrategia Irlanda, Israel y Eslovenia quedan totalmente fuera del análisis, mientras que Grecia y

\footnotetext{
${ }^{12}$ El índice de descentralización utilizado es la media de los niveles de decisión (1=central, 2=regional, $3=$ provincial, $4=$ local y $5=$ =escolar) ponderada por los porcentajes de decisiones educativas que se toman en cada nivel.

${ }^{13}$ Medido a través del porcentaje de alumnos repetidores en la secundaria obligatoria.

${ }^{14}$ El índice es igual a la media por países del índice de estatus socioeconómico y cultural de las familias de los estudiantes proporcionado por el estudio PISA. Dicho índice ha sido creado a partir del estatus ocupacional de los padres, de su nivel de educación y de la riqueza del hogar.
} 
Luxemburgo sólo aparecen en los análisis del primer grupo y Suiza y Polonia en los del segundo.

Los modelos de la segunda etapa estimados inicialmente para los 22 países, se han estimado de nuevo para los dos grupos de 17 países obteniendo resultados muy similares a los iniciales y confirmando la idoneidad de las variables individuales seleccionadas. El reducido número de unidades de segundo nivel no permite la especificación de modelos demasiado complejos, desaconsejando por tanto la utilización simultánea de varias variables contextuales en un mismo modelo. Por ello, en la tercera etapa se ha optado por proceder a la modelización de los coeficientes de las variables individuales mediante una única variable de segundo nivel en cada caso ${ }^{15}$.

\section{Resultados}

La opinión de los europeos sobre la educación y factores que inciden en su satisfacción

En líneas generales podemos decir que los europeos aprueban el estado de la educación en su país puesto que la nota media global para el conjunto de los 22 países incluidos en el estudio es 5,14. El gráfico 1 muestra los intervalos de confianza del 95\% para la estimación de las valoraciones medias de los diferentes países. Se ha incluido en el gráfico una línea horizontal en el valor 5 como referencia del centro de la escala de valoración de esta pregunta (de 0 a 10). El grafico muestra que, en promedio, los ciudadanos de 17 de los 22 países (un 77\%) aprueban el estado de la educación en su país. Sin embargo, existen diferencias importantes entre ellos. Cabe destacar en el extremo superior derecho Finlandia $(7,84)$ y Dinamarca $(7,32)$ con valoraciones por encima del 7. A continuación aparece un grupo de países con valoraciones por encima del 5,5 que comparten la característica de ser pequeños en extensión o en población. En

\footnotetext{
${ }^{15}$ El método de estimación seguido ha sido el de máxima verosimilitud. La aplicación de dicho método presupone la distribución normal univariante de los residuos individuales y multivariante de los efectos aleatorios de segundo nivel, así como una correcta especificación del modelo. La asociación lineal entre la variable respuesta y las variables explicativas se ha explorado previamente a la especificación del modelo. Las hipótesis distribucionales se han revisado a partir de los residuos, no encontrando evidencia suficiente para descartar las hipótesis de normalidad mencionadas.
} 
el otro extremo destaca Portugal con una valoración media de 3,74 y Grecia, Alemania, Israel y España con medias entre 4,5 y 5.

\section{Gráfico 1.}

\section{Valoración del estado de la educacion}

Intervalos de confianza al 95\% de las medias por paises

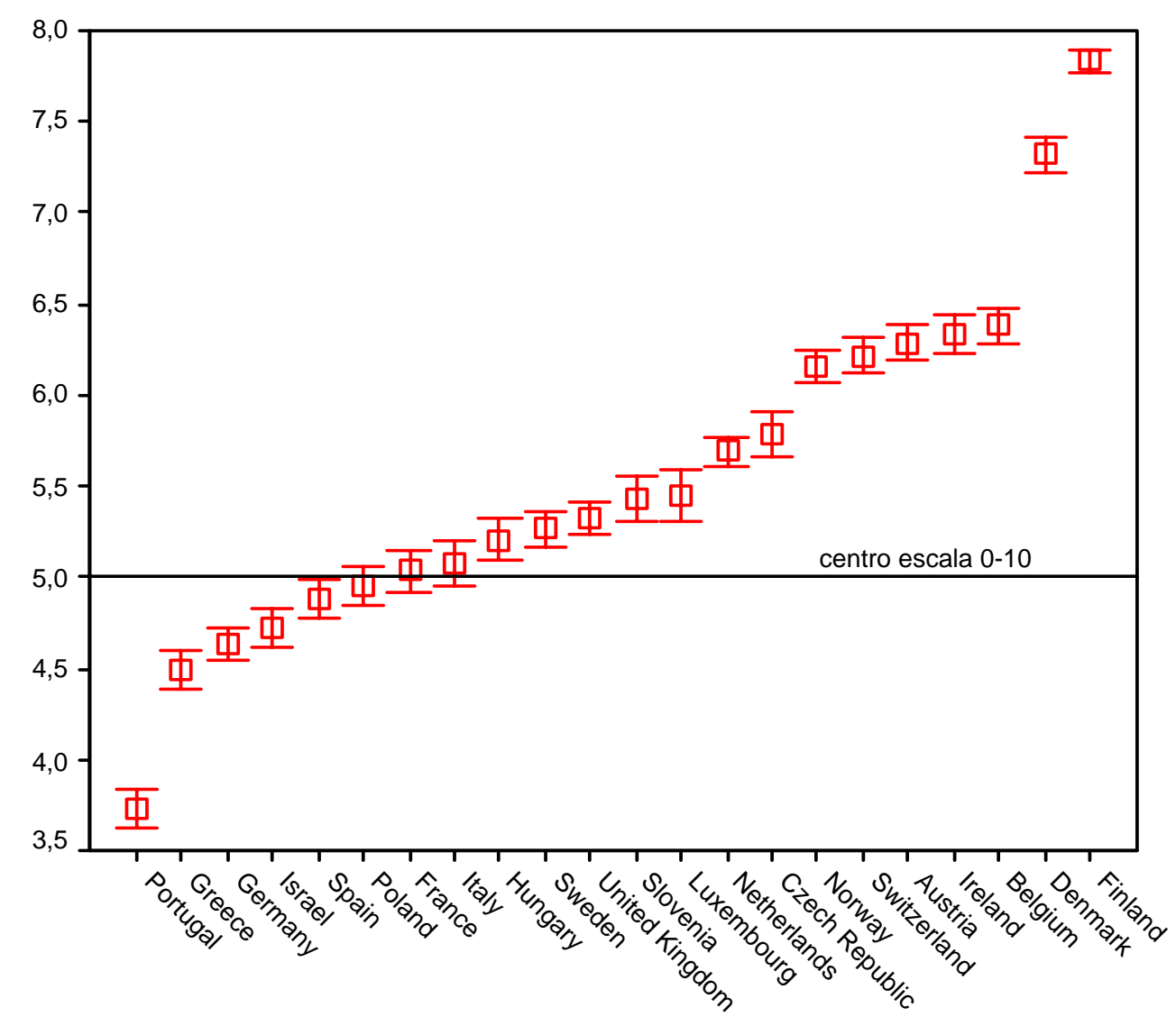

En términos generales y con algunas excepciones, estos datos parecen indicar que los ciudadanos de los países nórdicos y centroeuropeos están más satisfechos con la educación en su país que los de los países del sur de Europa. Además, un contraste de homogeneidad de las varianzas indica que en unos países la opinión es más homogénea que en otros. Entre los primeros cabe destacar Finlandia, Holanda y Dinamarca (con desviaciones estándar de 1,4, 1,8 y 1,8 respectivamente). Entre los países cuyos ciudadanos muestran opiniones más dispersas cabe destacar Israel $(2,7)$, Grecia $(2,6)$ y Luxemburgo $(2,5)$. 
El análisis descriptivo realizado ha puesto de manifiesto la existencia de diferencias importantes en la valoración media de la educación entre países, así como en la variabilidad de la opinión de sus respectivos ciudadanos sobre este tema. ¿A qué se debe esa valoración poco satisfactoria del estado de la educación en algunos países? Parece razonable aceptar que valoraciones medias negativas reflejan un estado de la educación en esos países inferior al que los ciudadanos desearían. La mayoría de los países incluidos en este estudio son miembros de la Unión Europea y, concretamente, los que ocupan las posiciones extremas, Grecia, Portugal y Alemania por un lado y Dinamarca y Finlandia por otro, son países fundadores o que se incorporaron hace casi veinte años. Cabría esperar que debido a las políticas de convergencia y a las ayudas económicas compensatorias, el estado de la educación hubiera alcanzado unos niveles de calidad semejantes que deberían verse reflejados en valoraciones más homogéneas. No sería de extrañar, en cambio, la presencia de diferencias en las opiniones de los ciudadanos de los países de reciente incorporación.

Tras unos análisis exploratorios, se descartaron las variables individuales y contextuales que no mostraban ninguna relación significativa con la satisfacción con la educación. Una vez obtenido el modelo explicativo de la satisfacción con la educación del país en función de variables individuales, se ha procedido a modelizar la ordenada en el origen a partir de variables contextuales. Las variables incluidas en los análisis que finalmente han mostrado tener un efecto significativo en la satisfacción con la educación son: el sexo, el nivel de estudios ${ }^{16}$ y la proximidad al gobierno, entre les variables individuales y el gasto público en educación, el grado de descentralización de las políticas educativas, el grado de inclusión del sistema y el estatus socioeconómico y cultural medio de la población entre las contextuales. La Tabla 1 resume la distribución por países de los valores de cada una de esas variables contextuales.

\footnotetext{
${ }^{16}$ El nivel de estudios alcanzado por el encuestado se ha introducido en el análisis a partir de la construcción de tres variables dummy correspondientes a no tener estudios, tener estudios secundarios y tener estudios terciarios, respectivamente, siendo la categoría de referencia estudios primarios.
} 
Tabla 1. Medias por países de la variable dependiente y de las variables contextuales.

\begin{tabular}{lccccc}
\hline País & $\begin{array}{c}\text { Satisfacción } \\
\text { con la } \\
\text { educación }\end{array}$ & $\begin{array}{c}\text { Gasto público } \\
\text { en educación }\end{array}$ & $\begin{array}{c}\text { Descentralización de } \\
\text { las decisiones } \\
\text { educativas }\end{array}$ & $\begin{array}{c}\text { Porcentaje de } \\
\text { repetidores }\end{array}$ & $\begin{array}{c}\text { Índice de estatus } \\
\text { socioeconómico y } \\
\text { cultural }\end{array}$ \\
\hline Austria & 6,3 & 5,8 & 3,1 & 4,7 & 0,1 \\
Alemania & 4,6 & 4,6 & 2,9 & 14,1 & 0,2 \\
Bélgica & 6,4 & 5,9 & 2,7 & 6,7 & 0,2 \\
Dinamarca & 7,3 & 8,4 & 3,9 & 0,7 & 0,2 \\
España & 4,9 & 4,4 & 2,3 & 25,2 & $-0,3$ \\
Finlandia & 7,8 & 6,3 & 4,2 & 0,0 & 0,3 \\
Francia & 5,0 & 5,7 & 2,0 & 26,7 & $-0,1$ \\
Grecia & 4,5 & 3,9 & 1,6 & 6,3 & $-0,2$ \\
Holanda & 5,7 & 5,0 & 5,0 & 9,5 & 0,1 \\
Hungría & 5,2 & 5,1 & 4,6 & 3,8 & $-0,1$ \\
Italia & 5,1 & 5,0 & 3,1 & 5,7 & $-0,1$ \\
Luxemburgo & 5,5 & 3,6 & 2,4 & 25,3 & - \\
Noruega & 6,2 & 7,2 & 3,5 & 0,0 & 0,6 \\
Polonia & 5,0 & 5,6 & - & 1,9 & $-0,2$ \\
Portugal & 3,7 & 5,9 & 2,6 & 16,9 & $-0,6$ \\
Reino Unido & 5,3 & 4,8 & 4,5 & 0,9 & - \\
República Checa & 5,8 & 4,4 & 4,4 & 1,7 & 0,2 \\
Suecia & 5,3 & 7,6 & 4,0 & 1,0 & 0,3 \\
Suiza & 6,2 & 5,6 & - & 8,2 & $-0,1$ \\
\hline Fiza & & & & &
\end{tabular}

Fuente: ESE 2002 y OECD 2004.

Tal como se puede observar, hay una gran concentración de valores de gasto público alrededor del valor central $(5,5)$. Destacan Dinamarca, Suecia, Israel y Noruega como los países que más invierten en educación, todos ellos con porcentajes superiores al 7\% del PIB. En el otro extremo se hallan Luxemburgo y Grecia, países que dedican a la educación menos de un 4\% del PIB. La asociación entre la inversión económica en educación y la satisfacción de los ciudadanos con la misma es positiva pero no muy intensa (coeficiente de correlación de Pearson =0,34).

Aunque con algunas excepciones, el índice de descentralización muestra que por lo que respecta a las decisiones educativas los países del sur de Europa son los más centralizados y los países nórdicos los más descentralizados. Los casos extremos son Holanda, que tiene la máxima descentralización (todas las decisiones educativas relativas a la educación secundaria se toman en los propios centros de enseñanza), y Grecia, que es donde hay una mayor centralización (un 80\% de las decisiones son tomadas por el gobierno central). Por lo que respecta a su relación con la satisfacción de 
los ciudadanos, los datos indican que niveles altos de descentralización se corresponden con valoraciones elevadas del estado de la educación (coeficiente de correlación= 0,43).

Francia, España y Luxemburgo destacan por tener un bajo nivel de inclusión en contraste con los países nórdicos y el Reino Unido, que presentan porcentajes de repetidores que no superan el uno por ciento. Parece claro que estos datos son el reflejo del tipo de sistema educativo vigente en cada país. Mientras que en los países nórdicos el sistema puede ser considerado claramente inclusivo, países como España y Francia han optado hasta ahora por una evaluación diferenciada por materias y por un ritmo en la progresión uniforme para todos los estudiantes con lo que es inevitable que una parte importante de los alumnos quede rezagada. Porcentajes elevados en el número de repetidores están asociados con valoraciones bajas de la educación por parte de los ciudadanos (coeficiente de correlación $=-0.49$ ).

En cuanto al estatus socioeconómico y cultural, también son los países del sur de Europa, especialmente Portugal los que tienen los valores medios más bajos y los países nórdicos, especialmente Noruega, los que los tiene más altos. En cuanto a su relación con la valoración de la educación por parte de los ciudadanos, es en los países con un elevado nivel socioeconómico y cultural donde hay mayor satisfacción con el estado de la educación (coeficiente de correlación $=0.63$ ).

\section{Efectos individuales}

La Tabla 2 presenta los resultados de la estimación de 6 modelos multinivel. Los datos de la parte superior son las estimaciones de los coeficientes de las variables individuales y contextuales así como de las interacciones entre variables de ambos niveles, mientras que los de la parte inferior son los componentes de la varianza para los modelos de dos niveles (individuos y países). Todos los valores son significativos al nivel del 0,05. 
Tabla 2. Efectos individuales y contextuales en la satisfacción con el estado de la educación (2002).

\begin{tabular}{|c|c|c|c|c|c|c|}
\hline & Modelo 1 & Modelo 2 & Modelo 3 & Modelo 4 & Modelo 5 & Modelo 6 \\
\hline & $\begin{array}{c}\text { Solo } \\
\text { variables } \\
\text { individuales }\end{array}$ & $\begin{array}{c}\text { Gasto } \\
\text { público en } \\
\text { educación }\end{array}$ & $\begin{array}{c}\text { Descentrali- } \\
\text { zación de las } \\
\text { decisiones } \\
\text { educativas }\end{array}$ & $\begin{array}{c}\text { Solo } \\
\text { variables } \\
\text { individuales }\end{array}$ & $\begin{array}{l}\text { Porcentaje } \\
\text { de } \\
\text { repetidores }\end{array}$ & $\begin{array}{c}\text { Índice de } \\
\text { estatus } \\
\text { socioeconómi } \\
\text { co y cultural }\end{array}$ \\
\hline \multicolumn{7}{|l|}{ Efectos fijos } \\
\hline constante & 4,31 & 1.51 & 2.23 & 4.42 & 4.87 & 4.38 \\
\hline Proximidad al gobierno & 0,32 & 0.53 & 0.47 & 0.31 & 0.31 & 0.31 \\
\hline Mujer & -0.05 & -0.41 & -0.05 & -0.06 & -0.06 & -0.06 \\
\hline Estudios secundarios & $-0,16$ & $-0,16$ & $-0,15$ & -0.17 & -0.17 & -0.17 \\
\hline Estudios terciarios & $-0,35$ & $-0,73$ & $-0,34$ & -0.36 & -0.36 & -0.36 \\
\hline Gasto público en educación & & 0.52 & & & & \\
\hline Descentralización & & & 0.64 & & & \\
\hline Porcentaje de repetidores & & & & & -0.05 & \\
\hline Índice socioeconómico y cultural & & & & & & 2.23 \\
\hline \multicolumn{7}{|l|}{ Interacciones: } \\
\hline Gasto público*Prox. al gobierno & & -0.04 & & & & \\
\hline Gasto público*Mujer & & 0.06 & & & & \\
\hline Gasto público*Estudios terc. & & 0.07 & & & & \\
\hline Descentraliz.* Prox. al gobierno & & & -0.05 & & & \\
\hline \multicolumn{7}{|l|}{$\begin{array}{l}\text { Efectos aleatorios (varianza } \\
\text { residual de los coeficientes) }\end{array}$} \\
\hline \multicolumn{7}{|l|}{ Nivel 2 (país) } \\
\hline Constante & 0,758 & 0.825 & 0.924 & 0.74 & 0.53 & 0.39 \\
\hline Proximidad al gobierno & & 0.005 & 0.005 & & & \\
\hline Mujer & & - & & & & \\
\hline Estudios terciarios & & 0.014 & & & & \\
\hline \multicolumn{7}{|l|}{ Nivel 1 (individuo) } \\
\hline Sigma cuadrado & 3.965 & 3.916 & 3.922 & 3.93 & 3.93 & 3.93 \\
\hline Coefic. Correlación intra países & 0.160 & & & 0.159 & & \\
\hline$-2 * \log ($ verosimilitud) & 122476 & 122166 & 122197 & 125066 & 125060 & 125055 \\
\hline $\mathrm{N}^{\circ}$ de parámetros estimados & 7 & 16 & 11 & 7 & 8 & 8 \\
\hline p-valor & & 0.000 & 0.000 & & 0.010 & 0.001 \\
\hline
\end{tabular}

Los valores son estimaciones de los coeficientes de nivel 1 (individuos) y nivel 2 (países) y de los componentes de las varianzas para seis modelos explicativos de la variable dependiente "Satisfacción con el estado de la educación en el propio país”. Todos los efectos, tanto los fijos como los aleatorios son significativos al nivel 0.05.

Los Modelos 1 y 4 tienen la misma especificación pero han sido estimado para dos grupos de países distintos, siendo sus resultados prácticamente iguales. Los signos de los coeficientes permiten extraer las siguientes conclusiones: 
- A mayor afinidad con el Gobierno, mayor satisfacción con el estado de la educación.

- $\quad$ Las mujeres se muestran más críticas que los hombres.

- Asimismo, los individuos que tienen estudios secundarios y terciarios son más críticos con el estado de la educación en su país que los que tienen estudios primarios, (coeficientes $-0,16$ y $-0,35$, respectivamente, en el modelo 1 ).

Por lo tanto, con independencia del grado de afinidad que un ciudadano pueda tener con su gobierno, la satisfacción con la educación es menor en las mujeres y en los que tienen un buen nivel de estudios que en el resto.

La introducción de estas variables individuales ha comportado una reducción apreciable en el error de predicción. Así para el primer grupo de países (modelo 1) la mejora en la predicción que supone la introducción de las variables individuales es del $14 \%{ }^{17}$ para las valoraciones individuales y del 23\% para las valoraciones medias de dichos países.

Tanto el Modelo 1 como el Modelo 4 (modelos ambos de descomposición de la varianza) muestran que un $16 \%$ de la variación total de las opiniones sobre el estado de la educación corresponde a diferencias entre países (coeficiente de correlación intra igual a 0,16), justificando la conveniencia de introducir variables de país que permitan explicar tales diferencias.

\section{Efectos contextuales (modelos para el primer grupo de países)}

Los modelos 2 y 3, estimados para el mismo grupo de países que el modelo 1, difieren en la variable de segundo nivel introducida en cada caso (gasto público en el primero y descentralización en el segundo).

\footnotetext{
${ }^{17}$ Para el cálculo de la reducción en los errores de predicción se han utilizado los indicadors propuestos por Snijders and Bosker (1999). Se han comparado, pues, las estimacions de las varianzas residuales entre países y entre individuos dentro de los países ( $\tau_{00}=0,758$ y $\sigma^{2}=3,965$, respectivamente) del modelo 1 con los respectivos valores del modelo nulo ( $\tau_{00}=0,987$ y $\left.\sigma^{2}=4,505\right)$. Las estimaciones para el modelo nulo o modelo sin variables explicativas no aparecen en la Tabla 2.
} 
La introducción de la variable gasto público en educación y la estimación de las interacciones de dicha variable con cada una de las tres variables individuales (Modelo 2) permite afirmar que:

- $\quad$ En los países que destinan un mayor presupuesto a la educación los ciudadanos muestran una mayor satisfacción con el estado de la educación.

- La interacción entre gasto público en educación y proximidad al gobierno reduce el impacto positivo de esta última variable en la opinión sobre el estado de la educación. En aquellos países en que el gasto público en educación es alto, la proximidad al gobierno no influye tanto en la opinión individual sobre el estado de la educación como en aquellos en los que el gasto público es menor.

- Un elevado volumen de gasto público reduce la visión mas crítica de las mujeres respecto de los hombres sobre el estado de la educación. En aquellos países donde el nivel de gasto público en educación es mayor, se observa una reducción de la diferencia de opinión entre géneros.

- Asimismo, la interacción entre gasto público en educación y nivel educativo individual alto reduce el impacto negativo de esta última variable en la opinión sobre el estado de la educación.

En el Modelo 3, que incorpora la variable índice de descentralización de las políticas educativas, únicamente ha resultado ser significativa una de las interacciones. Se trata de la interacción entre descentralización y proximidad al gobierno. La observación de los valores de los coeficientes estimados permite afirmar que:

- A mayor descentralización de las decisiones que afectan a la educación, mayor satisfacción con el estado de la educación.

- $\quad$ La interacción entre descentralización y proximidad al gobierno reduce el impacto positivo de esta última variable en la opinión sobre el estado de la educación. En los países con un alto grado de descentralización, la afinidad con el gobierno no tiene tanto efecto en la opinión individual sobre el estado de la educación como lo tiene en los países de gestión educativa centralizada. Se podría conjeturar que la mayor participación en las decisiones que involucran temas de educación conlleva que el ciudadano separe la opinión sobre el gobierno de la opinión sobre el estado de la educación. 
Los modelos 5 y 6, así como el modelo 4, se han estimado para el segundo grupo de países. En el Modelo 5 se ha introducido el porcentaje de repetidores en secundaria obligatoria, y en el Modelo 6 un índice de estatus socioeconómico y cultural medio de cada país. Ninguna de las interacciones analizadas entre estas variables y las tres características individuales, sexo, educación y proximidad al gobierno, ha resultado ser significativa. Los coeficientes estimados en ambos modelos indican que:

- Un porcentaje elevado de repetidores en la enseñanza secundaria tiene un efecto negativo en la valoración de los ciudadanos sobre el estado de la educación.

- Cuanto mayor es el nivel socioeconómico y cultural de un país, mayor es la satisfacción de sus ciudadanos con el estado de la educación en el mismo.

Tal como se desprende de la comparación del modelo 4 con los modelos 5 y 6 (véase la parte inferior derecha de la Tabla 2), el porcentaje de repetidores y el índice de estatus socioeconómico y cultural reducen la varianza residual entre países de 0,74 a 0,53 y a 0,39, respectivamente, lo cual representa una mejora en la predicción de las valoraciones medias de dichos países del 29\% cuando se introduce el porcentaje de repetidores y del $47 \%$ cuando la variable introducida es el índice de estatus socioeconómico y cultural.

\section{Conclusiones}

En este trabajo se ha planteado un modelo explicativo de la satisfacción de los ciudadanos con el estado de la educación en su país en el que, más allá de relacionar las variaciones de satisfacción con las características específicas de los individuos, lo que interesaba era analizar la influencia de las políticas educativas y otras características institucionales y sociales de cada país en dicha satisfacción. El análisis de los datos del año $2002^{18}$ de los países incluidos en la Encuesta Social Europea ha permitido

\footnotetext{
${ }^{18}$ Y también del 2004 (véase Anexo 1).
} 
contrastar el conjunto de hipótesis que se habían planteado, tanto las de tipo individual como las de tipo contextual.

Por lo que respecta a las hipótesis sobre efectos individuales, han sido contrastadas positivamente tanto la que hace referencia a la diferencia de valoraciones entre sexos como la que hace referencia a la diferencia de valoraciones según nivel de estudios, habiéndose confirmado que las mujeres están menos satisfechas con la educación que los hombres y que los ciudadanos con un nivel educativo alto son más críticos que los que tienen un nivel educativo medio y éstos, a su vez, más críticos que los que tienen un nivel educativo bajo. Sin embargo, no ha sido posible confirmar las hipótesis que planteaban diferencias según edad.

Asimismo, ha resultado significativa y tener un efecto positivo en la valoración de la educación la variable de proximidad al gobierno, que se había introducido como variable de control. Es decir, los datos parecen confirmar que la proximidad al partido del gobierno influye positivamente en la valoración de la educación. Pero los datos confirman también que, sea cual sea su grado de afinidad con el gobierno, las mujeres dan valoraciones inferiores que las de los hombres y que las personas con mayor nivel de estudios dan valoraciones inferiores que las de menor nivel de estudios.

Por lo que respecta a las variables contextuales, los datos aportan evidencia empírica a favor de la importancia de los recursos económicos en las valoraciones sobre la educación. Así, en aquellos países donde se invierten más recursos en educación, la satisfacción de la ciudadanía es más elevada. Pero es que, además, un presupuesto elevado destinado a educación reduce las diferencias de percepción individual entre sexos y entre individuos con diferentes niveles de estudios: a más presupuesto, menor visión crítica de las mujeres respecto los hombres y menor visión crítica de los ciudadanos con nivel alto de estudios respecto los de nivel bajo. También es importante señalar que a mayor volumen de recursos destinados a educación, menor es la influencia de la proximidad al partido gobernante en la valoración de la educación.

Otra de las hipótesis planteadas hacía referencia al nivel de descentralización de las políticas educativas, considerando que cuanto mayor fuera el nivel de descentralización más satisfecho debería estar el ciudadano. También esa hipótesis ha sido contrastada 
positivamente. Según el análisis realizado, a mayor descentralización de las decisiones en materia educativa en los países, mejor es la valoración de la educación por parte de sus ciudadanos. También aquí es importante señalar que un elevado grado de descentralización mitiga el efecto de la sintonía con el gobierno en la evaluación de la educación.

Una tercera hipótesis que ha sido contrastada positivamente es la que hace referencia al grado de inclusión del sistema educativo. Se suponía que un sistema que presentara múltiples barreras que los alumnos deberían superar para progresar en sus estudios, y que obligara a buena parte de ellos a repetir cursos o a abandonar, debía producir poca satisfacción en la ciudadanía. Los datos muestran que, efectivamente, en los países con sistemas poco inclusivos las valoraciones sobre la educación son inferiores que en los países con sistemas más inclusivos.

Finalmente, ha podido ser también aceptada una cuarta hipótesis referida a características del contexto. Se trata de la que sostiene que un entorno socioeconómico y cultural elevado influye positivamente en los resultados de la educación y ello debe reflejarse en las percepciones ciudadanas. La introducción de un índice de estatus socioeconómico y cultural medio de los países en el análisis ha permitido comprobar la validez de dicha hipótesis: cuanto mayor es el nivel socioeconómico y cultural de un país, más satisfechos están sus ciudadanos con la educación.

Los datos no han permitido confirmar, sin embargo, que las condiciones de trabajo y el prestigio social del profesorado, así como la participación e implicación del alumnado sean aspectos que influyen en la opinión de los ciudadanos sobre el estado de la educación. Probablemente en algunos casos ello sea debido a que no se ha podido disponer de buenos indicadores y en otros a la escasez del número de países que han podido ser incluidos en el análisis. En definitiva, del conjunto de resultados obtenidos en el estudio parece desprenderse la siguiente conclusión:

La satisfacción con la educación será más alta en aquellos países que inviertan en ella un elevado volumen de recursos económicos, que tengan un sistema educativo más inclusivo, que dispongan de un sistema de toma de decisiones educativas más 
descentralizado, y cuyas poblaciones tengan un nivel socioeconómico y cultural más elevado.

\section{Bibliografía}

Blekesaune, M. y Quadagno, J. (2003). Public Attitudes towards Welfare Satte Policies: A comparative Analysis of 24 Nations, European Sociological Review 19: 415-27.

Bonal, X. 1998. "La política educativa: dimensiones de un proceso de transformación (1976-1996)” en Gomà y Subirats (coords.) Políticas públicas en España. Contenidos, redes de actores y niveles de gobierno. Barcelona: Ariel.

Consell Superior d'Avaluació del Sistema Educatiu (2005) El sistema educatiu finlandès vist des de Finlàndia. Breus apunts sobre el sistema educatiu finlandès $i$ català. Barcelona: Generalitat de Catalunya.

Cuxart, A. y C. Riba (2005). "Aspectos metodológicos de la Encuesta Social Europea" en Torcal, M., L. Morales y S. Pérez-Nievas (eds): España: sociedad y política en perspectiva comparada. Valencia: Tirant lo Blanch, 21-40.

Dalton, R.J. (1996, 2nd ed.). Citizens Politics. Public Opinion and Political Parties in Advanced Industrial Democracies. New Jersey: Chatham House.

Delors, J. (coord.) (1996) La Educación encierra un tesoro. Pistas y Recomendaciones. Informe para la UNESCO de la Comisión Internacional sobre Educación en el siglo XXI.

Fraile, M. (2005). "Evaluación de políticas públicas y preferencias sobre nivel de gobierno” en Torcal, Morales y Perez-Nievas (Eds) España : sociedada y política en perspectiva comparada, pag. 113-132, Valencia: Tirant lo Blanch.

Gairin, J. (coord.) (2005). La descentralización educativa: ¿Una solución o un problema? Barcelona: Cisspraxis.

Kish, L. (1965) Survey Sampling, New York: John Wiley and Sons.

Lazarsfeld, P.F., B. Berelson and H. Gaudet (1944) The People's Choice: How the Voters Makes up his Mind in a Presidential Campaign. New York: Duell, Sloan \& Pearce.

Llera, F.J. (2003) “La opinión pública: La diversidad de una nación plural” en Subirats y Gallego (eds) Veinte años de autonomías en España. Leyes, políticas públicas, instituciones y opinión pública, pág. 321-376. Madrid: CIS.

OECD (2004) Education at a Glance. OECD indicators 2004. OECD Publishing (http://www.oecd.org/edu/eag2004) 
OECD (2004) Learning for Tomorrow's World- First Results from PISA 2003. OECD Publishing (www.pisa.oecd.org).

Ortún, V. y G. López-Sasanova. (2002). The Spanish health system, capitation financing, incentives in health care management Documentos de Trabajo 3. Fundación BBVA.

Page, B. I. (1996) Who deliberates? mass media in modern democracy. Chicago: University Press.

Page, B.I y R. Shapiro. (1983) Effects of Public Opinion on Policies. American Political Science Review, 77 (1), 175-190.

Page, B.I y R.Y. Shapiro. (1992) The Rational Public. Chicago: University of Chicago Press.

Pallarès, F. y J. Gifreu (coord.) (1998) Comunicació política i comportament electoral. Barcelona: Editorial Mediterrània.

Riba, C. y A. Cuxart (2003). "Construyendo las bases para una comparación fiable: la Encuesta Social Europea 2002 en España”, Revista Española de Ciencia Política, N. 8: 165-185

Saris, W. E., A. Satorra and G. Coenders (2004) "A new Approach to Evaluating the Quality of measurement instruments: the split-ballot MTMM design. Sociological Methodology (34), 311-347.

Saris, W. E., W. vander Veld and I. Gallhofer (2004) "Development and improvement of questionnaires using predictions of reliability and validity" en Presser et al. (eds.) Methods for testing and evaluating survey questionnaires. Hooboken: Wiley, 275-299.

Schlesinger, M. and C. Heldman (2001) “ Gender Gap or Gender Gaps? New Perspectives on Support for Government Action and Policies” The Journal of Politics 63 (1), 59-92.

Snijders, T., and Bosker, R. (1999) Multilevel Analysis: An introduction to basic and advanced multilevel modeling. London: Sage Publications.

Squire, P. (1988) "Why the 1936 Literary Digest Poll Failed”, The Public Opinion Quartely, Vol. 52, No. 1, 125-133.

Stimson, J., M. MacKuen y R. Erikson. (1995). Dynamic Representation. American Political Science Review,89 (3), 543-565.

Subirats, J. (2004) ”Descentralización y coordinación en el estado autonómico: la política educativa”, en Planas, Subirats, Riba y Bonal La escuela y la nueva ordenación del territorio, pág. 65-103. Barcelona: Octaedro.

Subirats, J. (coord.) 2002. Gobierno Local y Educación: la importancia del territorio y la comunidad en el papel de la escuela. Barcelona: Ariel. 
Zaller, John (1991) Information, Values and Opinion. The American Political Science Review Vol 85 (4), 1215-1237.

\section{Anexo 1. Análisis para los datos de $2004^{19}$}

Las percepciones sobre la educación en 2002 y 2004

Un primer análisis descriptivo de los datos de la ESE de 2004 muestra diferencias significativas $^{20}$ respecto del 2002 en la percepción de la calidad de la educación en algunos países (Gráfico 2).

\section{Gráfico 2}

\section{Variación en la percepción de la educación. Media del 2004 menos media del 2002}

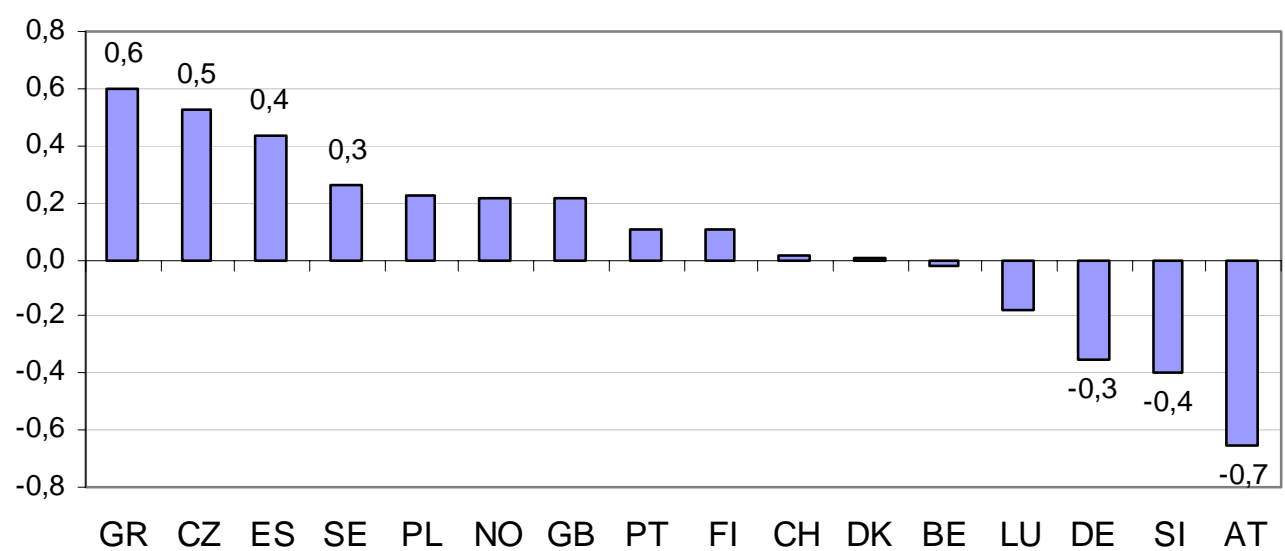

Los países en los que la valoración de la educación es claramente mejor en 2004 que dos años atrás son Grecia, República Checa y España, ésta última con una mejora de 0,44 puntos en una escala de 0 a 10 . El dato es importante porque Grecia y España habían recibido valoraciones negativas (inferiores a 5) en 2002 y en 2004 llegan al aprobado. Sin embargo, ése no es el caso de otros dos países que estaban a la cola de las

${ }^{19}$ En el momento de cerrar este trabajo solamente se disponía de datos de 17 de los 26 países participantes en la encuesta de 2004. Francia, Hungría, Italia y Holanda, que participaron en la ola 2002, todavía no habían hecho públicos sus datos.

${ }^{20}$ Para un nivel de significación del 5\% (margen de error como máximo de 0,2). 
valoraciones en 2002: la percepción sobre la calidad de la educación en Portugal no parece haber variado, mientras que en Alemania ha empeorado.

\section{Replicación para 2004 del análisis multinivel de 2002}

Debido a la no disponibilidad de los datos para la totalidad de los países, cada uno de los dos grupos de países para los que se estimaron los modelos de la tabla 2, se ha visto reducido a 13 países al combinar la información individual con la agregada.

La tabla 3 muestra los resultados de las estimaciones de los seis modelos multinivel de predicción de la satisfacción con la educación (tres para el primer grupo de países y 3 para el segundo), con la misma especificación que los modelos que se estimaron para los datos del 2002 y cuyos resultados se muestran en la tabla 2.

Tal como puede comprobarse, los resultados para los datos del 2004 son coherentes con los hallados en 2002. Existen pequeñas diferencias, atribuibles en parte a la no presencia en los análisis del 2004 de los datos de varios países presentes en los análisis del 2002 y a las dificultades de estimación de un modelo multinivel con un número tan escaso de países. Estas diferencias consisten en la pérdida de significación de la variable mujer en algunos de los modelos y la significatividad de la variable sin estudios en 2004 cuando no la tenía en 2002. Al margen de esas pequeñas excepciones, las demás estimaciones son muy similares en las dos tablas, tanto los signos y las magnitudes de los coeficientes como los valores de las varianzas.

Se puede concluir, por lo tanto, que los resultados del estudio obtenidos con datos de 2002 siguen vigentes en 2004 . 
Tabla 3. Efectos individuales y contextuales en la satisfacción con el estado de la educación (2004).

\begin{tabular}{|c|c|c|c|c|c|c|}
\hline & Modelo 1 & Modelo 2 & Modelo 3 & Modelo 4 & Modelo 5 & Modelo 6 \\
\hline & $\begin{array}{c}\text { Solo } \\
\text { variables } \\
\text { individuales }\end{array}$ & $\begin{array}{l}\text { Gasto } \\
\text { público en } \\
\text { educación }\end{array}$ & $\begin{array}{l}\text { Descentrali- } \\
\text { zación de las } \\
\text { decisiones } \\
\text { educativas }\end{array}$ & $\begin{array}{c}\text { Solo } \\
\text { variables } \\
\text { individuales }\end{array}$ & $\begin{array}{l}\text { Porcentaje } \\
\text { de } \\
\text { repetidores }\end{array}$ & $\begin{array}{l}\text { Índice de } \\
\text { estatus } \\
\text { socioeconómi } \\
\text { co y cultural }\end{array}$ \\
\hline $\begin{array}{l}\text { Efectos fijos } \\
\text { constante }\end{array}$ & $4.34 *$ & 1.64 & 1.67 & $4.52 *$ & $5.10 *$ & $4.45^{*}$ \\
\hline Proximidad al gobierno & $0.33 *$ & $0.51 *$ & $0.44^{*}$ & $0.32 *$ & $0.32 *$ & $0.32 *$ \\
\hline $\begin{array}{l}\text { Mujer } \\
\text { Sin estudios } \\
\text { Estudios secundarios } \\
\text { Estudios primarios }\end{array}$ & $\begin{array}{c}0.04 \\
0.18^{*} \\
-0.16^{*} \\
-0.32^{*}\end{array}$ & $\begin{array}{l}-0.23^{*} \\
0.17^{*} \\
-0.15^{*} \\
-0.86^{*}\end{array}$ & $\begin{array}{c}0.04 \\
0.18^{*} \\
-0.16^{*} \\
0.31^{*}\end{array}$ & $\begin{array}{c}0.03 \\
0.17^{*} \\
-0.16^{*} \\
-0.32^{*}\end{array}$ & $\begin{array}{c}0.03 \\
0.17^{*} \\
-0.16^{*} \\
-0.32^{*}\end{array}$ & $\begin{array}{c}0.03 \\
0.17^{*} \\
-0.16^{*} \\
-0.32^{*}\end{array}$ \\
\hline $\begin{array}{l}\text { Gasto público en educación } \\
\text { Descentralización } \\
\text { Porcentaje de repetidores } \\
\text { Índice socioeconómico y cultural }\end{array}$ & & $0.43^{*}$ & $0.84 *$ & & $-0.09 *$ & $1.90 *$ \\
\hline $\begin{array}{l}\text { Interacciones: } \\
\text { Gasto público*Prox. al gobierno } \\
\text { Gasto público*Mujer } \\
\text { Gasto público*Estudios terc. } \\
\text { Descentraliz.* Prox. al gobierno }\end{array}$ & & $\begin{array}{l}-0.03^{*} \\
0.05^{*} \\
0.09^{*}\end{array}$ & -0.03 & & & \\
\hline $\begin{array}{l}\text { Efectos aleatorios (varianza } \\
\text { residual de los coeficientes) }\end{array}$ & & & & & & \\
\hline $\begin{array}{l}\text { Nivel } 2 \text { (país) } \\
\text { Constante } \\
\text { Proximidad al gobierno } \\
\text { Estudios terciarios }\end{array}$ & 0.860 & $\begin{array}{l}1.058 \\
0.004 \\
0.006\end{array}$ & $\begin{array}{l}1.102 \\
0.005\end{array}$ & 0.782 & 0.378 & 0.463 \\
\hline $\begin{array}{l}\text { Nivel } 1 \text { (individuo) } \\
\text { Sigma cuadrado } \\
\text { Coefic. Correlación intra países }\end{array}$ & $\begin{array}{l}3.721 \\
0.188\end{array}$ & 3.693 & 3.698 & $\begin{array}{l}3.730 \\
0.173\end{array}$ & 3.730 & 3.730 \\
\hline $\begin{array}{l}-2 * \log \text { (verosimilitud) } \\
\mathrm{N}^{\mathrm{o}} \text { de parámetros estimados } \\
\text { p-valor }\end{array}$ & $\begin{array}{c}102134 \\
8\end{array}$ & $\begin{array}{c}101966 \\
17 \\
0.000\end{array}$ & $\begin{array}{c}101991 \\
12 \\
0.000\end{array}$ & $\begin{array}{c}103647 \\
8\end{array}$ & $\begin{array}{c}103638 \\
9 \\
0.003\end{array}$ & $\begin{array}{c}103640 \\
9 \\
0.008\end{array}$ \\
\hline
\end{tabular}

* indica los efectos fijos que son significativos al nivel 0.05. Todos los efectos aleatorios son significativos para este nivel. 